DOI: $10.12731 / 2306-1561-2013-4-18$

\title{
STUDY OF THE AUTOMATIC CONTROL SYSTEM OF THERMOELECTRIC OBJECT
}

\author{
Nikolaev A.B., Ni Zaw
}

\section{Abstract}

Thermoelectric phenomena - a collection of physical phenomena, which include three reversible thermoelectric effects: the Seebeck, Peltier and Thomson. These effects are associated with mutual conversion of heat energy into electric current in metals and semiconductors. Currently widely adopted the so-called thermoelectric effects in semiconductor technology based on the use of the Peltier effect. The essence of the Peltier effect is the conversion of the potential difference in the temperature difference. In this paper, we study the automatic control system (ACS) in a thermoelectric object.

Keywords: thermoelectric facility, the regulatory system, the transfer function, the perturbation, the transition process.

\section{УДК 681.518}

\section{ИССЛЕДОВАНИЕ СИСТЕМЫ АВТОМАТИЧЕСКОГО УПРАВЛЕНИЯ ТЕРМОЭЛЕКТРИЧЕСКИМ ОБЬЕКТОМ}

\author{
Николаев А.Б., Ни Зо
}

\section{Аннотация}

Термоэлектрические явления - это совокупность физических явлений, к числу которых относятся три обратимых термоэлектрических эффекта: Зеебека, Пельтье и Томсона. Эти эффекты связаны с взаимным превращением тепловой энергии в энергию электрического тока в металлах и полупроводниках. В настоящее время широкое применение получили так называемые термоэлектрические эффекты в полупроводниковой технике, основанные на использовании эффекта Пельтье. Суть эффекта Пельтье заключается в преобразовании разности потенциалов в разность температур. В данной статье проводится исследование системы автоматического управления (САУ) таким термоэлектрическим объектом.

Ключевые слова: термоэлектрический объект, система регулирования, передаточная функция, возмущение, переходной процесс. 


\section{Математическое описание системы автоматического управления термоэлектрическим объектом}

Для анализа и синтеза системы автоматического управления (САУ) необходимо иметь математическое описание объекта управления. Аналитическое определение динамических свойств объекта управления по конструктивным и физическим данным часто является сложной задачей. В таких случаях обращаются к идентификации объекта по экспериментальным данным [1, 4, 5]. В данной статье проведена идентификация объекта по временным характеристикам, а именно, по реакции объекта на скачкообразный входной сигнал $h(t)$. Обработка исходных данных эксперимента проводилась с помощью электронной таблицы MS Excel. Результаты расчета в графическом виде представлены на рисунке 1.

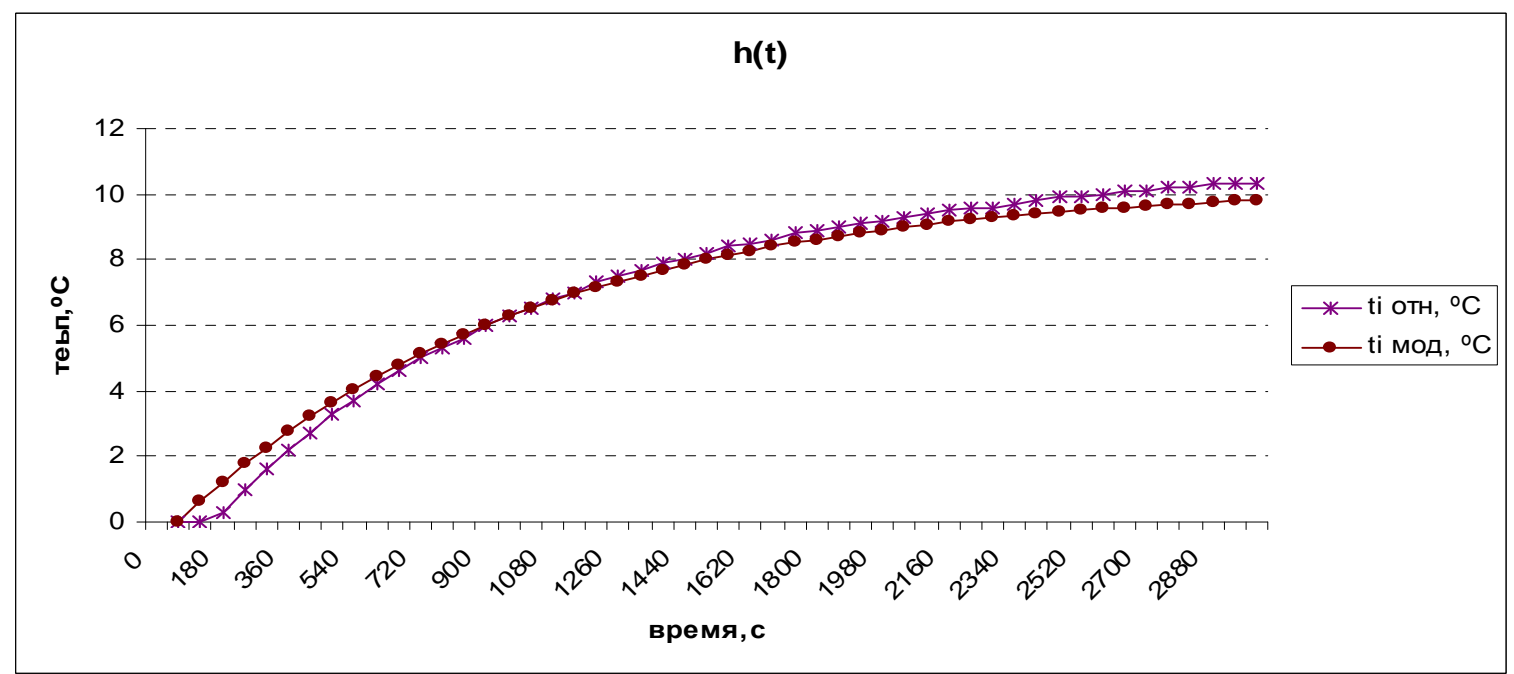

Рисунок 1 - Графическое изображение результатов h(t)

Экспериментальные переходные характеристики объекта $h(t)$ с достаточной точностью можно аппроксимировать экспоненциальной зависимостью вида: $h(t)=k_{0}\left(1-\exp \left(-t / T_{0}\right)\right)$, где $k_{0}-$ коэффициент передачи, $T_{0}-$ постоянная времени объекта.

Такая временная характеристика соответствует линейной математической модели в виде передаточной функции типового апериодического (инерционного) звена: $W_{0}(p)=\frac{K_{0}}{1+T_{0}} \mathrm{c}$ достаточно большой инерционностью $T_{0}=500-2000$, которую можно оценить моментом времени с координатой $h\left(T_{0}\right)=0.63 h_{y c m}$, где $h_{y c m}-$ установившееся значение $h(t)$ при $t \rightarrow \infty$. По экспериментальным данным определено $K_{0}=10,3 \mathrm{~K} / B$. Постоянная времени $T_{0}=960 \mathrm{c}$ (см. рисунок 1$)$.

Такая временная характеристика соответствует линейной математической модели в виде передаточной функции типового апериодического звена. Расхождения между экспериментальными данными $h(t)$ и теоретическими, определенными по модели, не 
превышают 3.5\%. Полученная модель использована при анализе системы автоматического управления этим объектом.

\section{Микропроцессорная система управления}

Для управления современным термическим оборудованием используются микропроцессорные системы управления (МПСУ). Такая система с точки зрения теории автоматического управления представляет собой классическую систему регулирования с контуром отрицательной обратной связи [2]. Структурная схема такой системы представлена на рисунке 2. где блок задания (Б3), схема сравнения (СС) и устройство управления (УУ) реализованы программно на однокристальной микроЭВМ. Исполнительное устройство (ИУ) и объект управления (ОУ) - это в типовом случае термическое оборудование. (Дт) - это датчик температуры. Передаточная функция разомкнутой такой системы регулирования имеет вид:

$$
\begin{aligned}
& W(p)=W_{\text {уу }}(p) \cdot W_{\text {иу }}(p) \cdot W_{\text {ооу }}(p) \cdot W_{\text {дат }}(p)
\end{aligned}
$$

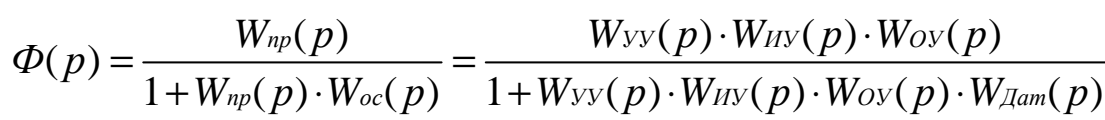

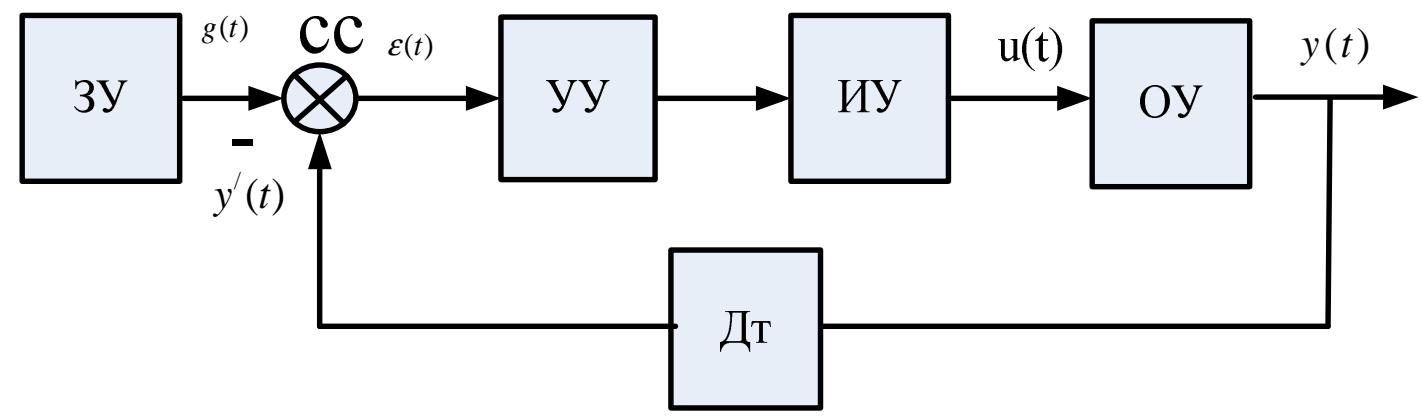

\section{Рисунок 2 - Структурная схема системы управления термическим оборудованием}

Математическая модель объекта управления может быть представлена согласно предыдущему пункту типовым апериодическим звеном с передаточной функцией $W_{O B}(p)=\frac{K_{O}}{1+T_{O} \cdot p}$ с указанными параметрами КО и ТО. Датчик температуры также может быть представлен апериодическим звеном, но с малой инерционностью порядка $T_{\text {Дат }} \approx 20$ с для терморезисторов и $T_{\text {Дат }} \approx 2 c$ для термопар. Передаточная функция регулятора в случае использования классических линейных законов регулирования имеет вид [3]:

Для П-закона

$$
W(p)=\frac{K_{\Pi} \cdot K}{\left(1+T_{O} \cdot p\right) \cdot\left(1+T_{\text {Дат }} \cdot p\right)}
$$

Для И-закона 


$$
W(p)=\frac{K_{u} \cdot K}{p\left(1+T_{o} p\right)\left(1+T_{\text {Даm }} p\right)}
$$

Для ПИ-закона

$$
W(p)=\left(K_{\Pi}+\frac{1}{T_{и} \cdot p}\right) \cdot \frac{K}{\left(1+T_{O} \cdot p\right) \cdot\left(1+T_{\text {Дат }} \cdot p\right)}
$$

Для ПИД-закона

$$
W(p)=\left(K_{\Pi}+\frac{1}{T_{и} \cdot p}+T_{\text {д }} \cdot p\right) \cdot \frac{K}{\left(1+T_{o} \cdot p\right) \cdot\left(1+T_{\text {Дат }} \cdot p\right)}
$$

\section{Реализация и экспериментальное исследование}

Математическое описание анализируемой системы дает возможность использовать специализированные программные пакеты (ПП) CALLISTO и MODOS при оценке значений оптимальных параметров настройки $T_{\Pi}$ - постоянная времени пропорциональности, $T_{И}$ - постоянная времени интегрирования, $T_{Д}$ - постоянная времени дифференцирования микропроцессорных блоков управления для различных модификаций термических приборов. Рассмотрим схему моделирования системы управления термическим оборудованием с ПИ-законом. По переходной характеристике исследуемой системы определим показатели качества. Результаты компьютерного моделирования системы управления термоэлектрическим объектом показаны на рисунке 3, а переходные процессы в САУ термоэлектрическим объектом с ПИрегулятором показаны на рисунке 4 (результаты исследования значений $t_{p}=47 c, \sigma=$ $\left.1,5 \%, \varepsilon_{y c m}=0\right)$.

Было проведено исследование влияния типовых возмущений на качество исследуемой САУ. Соответствующая структурная схема САУ показана на рисунке 5. Здесь же приведены основные теоретические соотношения для установившихся ошибок от полезного сигнала $X(t)$ и от возмущений $\left(f_{1}\right)$ и $\left(f_{2}\right)$. В САУ термоэлектрическим обьектом возмущения могут быть вызваны изменением температуры окружающей среды, изменением температуры теплопроводности, теплопередачи и теплообменник, несовершенством конструкции термоэлектрического полупроводникового вещества и т.п. 


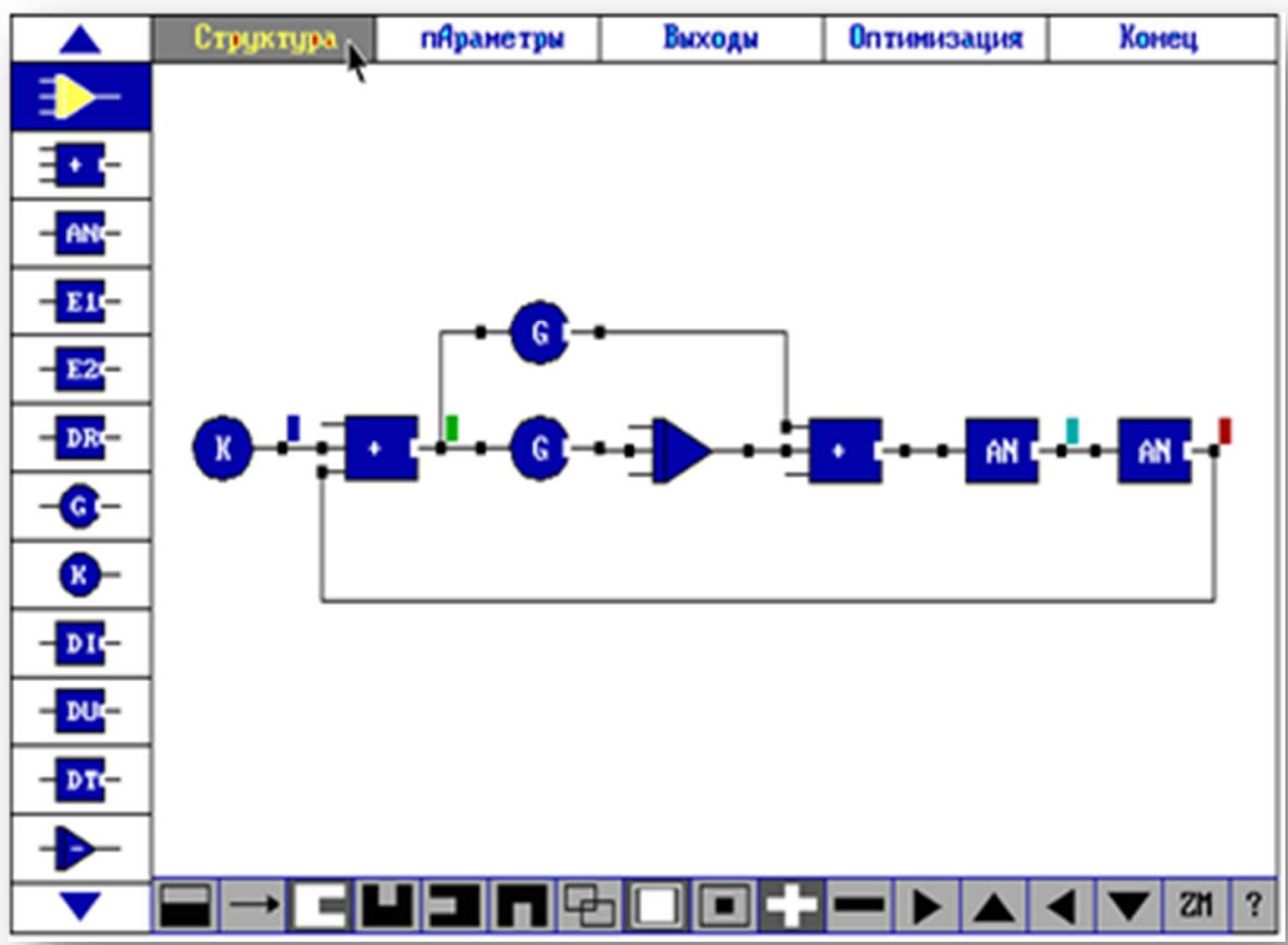

Рисунок 3 - Схема моделирования системы управления термоэлектрическим объектом с ПИ-регулятором

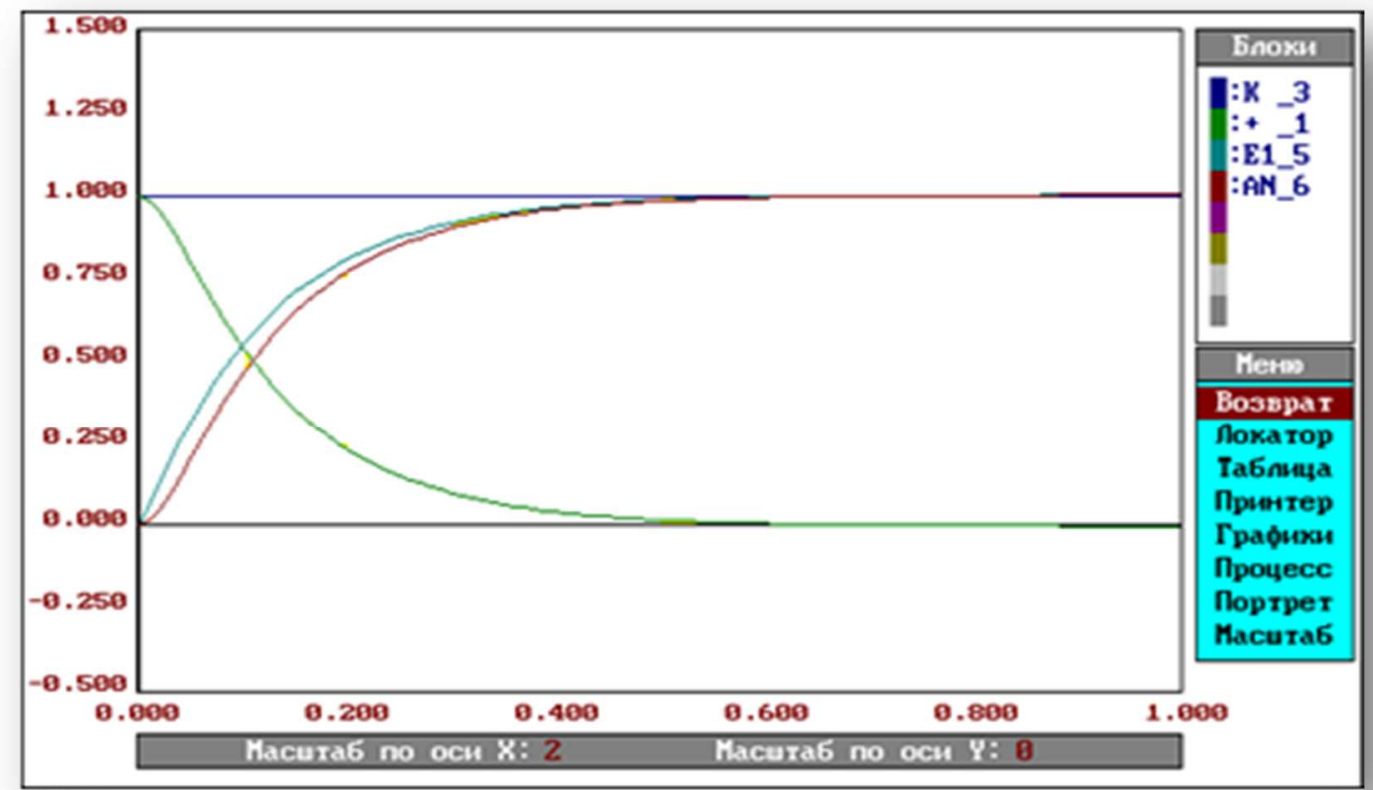

Рисунок 4 - Переходные процессы в САУ термоэлектрическим объектом с ПИрегулятором 


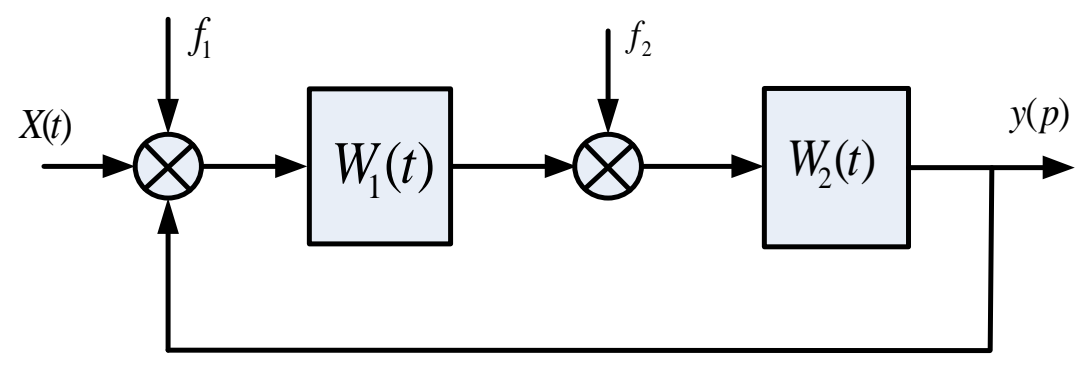

\section{Рисунок 5 - Структурная схема САУ в установившемся режиме в условиях воздействия возмущений}

Передаточная функция ошибки от полезного сигнала имеет вид:

$$
\begin{aligned}
& \Phi_{\varepsilon x}(p)=\frac{\varepsilon_{x}(p)}{x(p)}=\frac{x(p)-y(p)}{x(p)}=1-\frac{y(p)}{x(p)}=1-\Phi(p)=1-\frac{W(p)}{1+W(p)}=\frac{1+W(p)-W(p)}{1+W(p)}=\frac{1}{1+W(p)} \\
& \varepsilon_{y c m}=\varepsilon_{x y c m}+\varepsilon_{f_{1} y c m}+\varepsilon_{f_{2} y c m} \\
& \varepsilon_{x y c m}=\lim _{p \rightarrow 0} p \cdot X(p) \cdot \varphi_{\varepsilon x}(p) \\
& \varepsilon_{f_{1} y c m}=\lim _{p \rightarrow 0} p \cdot f_{1}(p) \cdot \varphi_{\varepsilon f_{1}}(p) \\
& \varepsilon_{f_{2} y c m}=\lim _{p \rightarrow 0} p \cdot f_{2}(p) \cdot \varphi_{\varepsilon f_{2}}(p)
\end{aligned}
$$

Возмущениея могут быть детерминированными (определенными во времени) и случайными. Влияние возмущений на качество системы может быть оценено с помощью передаточных функций ощибок. Поскольку в линейных системах справедлив принцип суперпозиции, то мы рассмотрим отдельно оценки ошибок в установившемся режиме. В исследуемой САУ возмущения могут быть приложены как ко входу САУ $\left(f_{1}\right)$, так и ко входу объекта управления $\left(f_{2}\right)$. Рассмотрим их влияние на точность САУ в установившемся режиме при различных законах управления. Схема моделирования САУ для П-закона и ПИ-закона представлены на рисунке 6.

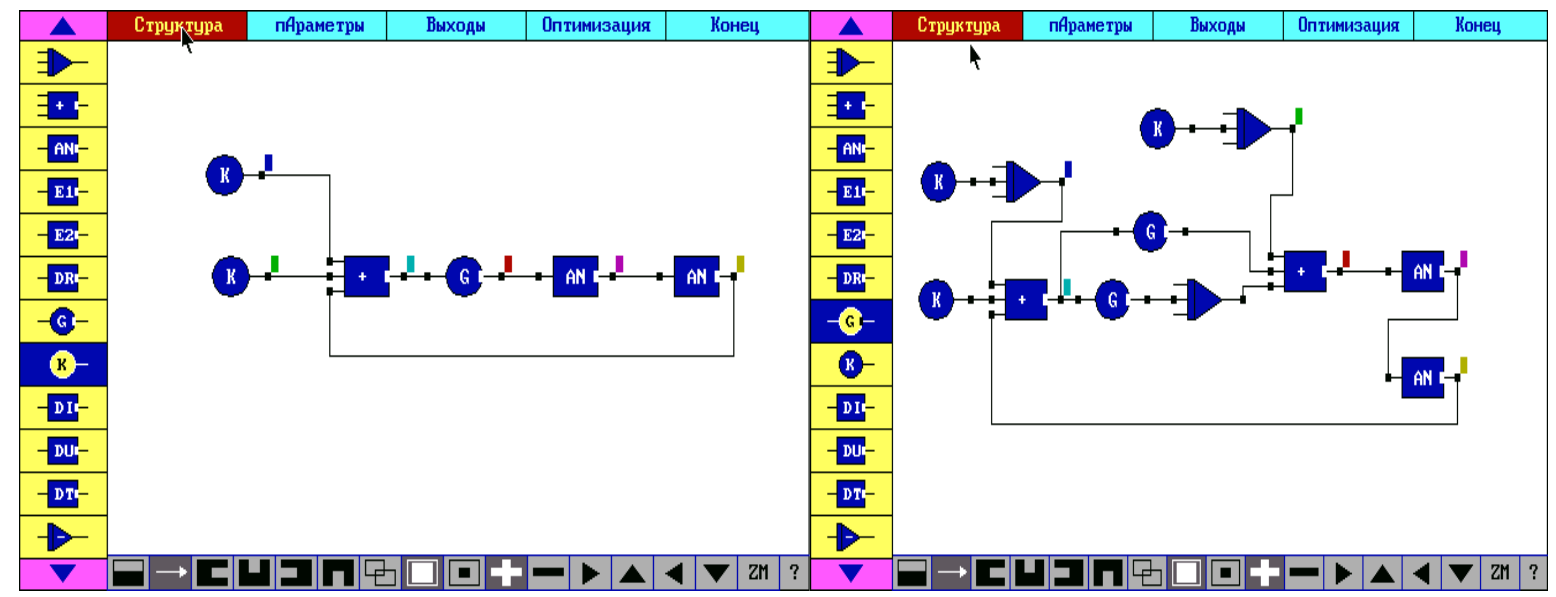

Рисунок 6 - Схемы моделирования системы управления термическим объектом с П и ПИ-законами 
Результаты компьютерного моделирования подтвердили теоретический вывод о том, что возмущение $f_{2}(t)=a_{2} t$ входит в общую ошибку $\varepsilon_{y c m}$, и показали, что такое воздействие на входе САУ дает ошибку, равную $a_{2} / K_{h}$. Полученные результаты по исследованию влияния возмущений на точность САУ представлены в таблице 1.

Таблица 1 - Результаты исследования влияния возмущений на точность САУ

\begin{tabular}{|l|l|c|c|c|c|}
\hline \multirow{2}{*}{$\begin{array}{l}\text { № } \\
\text { Тип закона } \\
\text { управления }\end{array}$} & \multicolumn{2}{|c|}{$f_{1}(t)$} & \multicolumn{2}{c|}{$f_{2}(t)$} \\
\cline { 2 - 6 } & $a_{2} .1(t)$ & $a_{1} .1(t)$ & $a_{1} . t$ & $a_{2} . t$ \\
\hline 1 & П-закон & $\varepsilon_{f 1 y c m} \cong a_{1}$ & $\varepsilon_{f 1 y c m} \cong \infty$ & $\varepsilon_{f 2 y c m}=\frac{a_{2}}{K_{\Pi}}$ & $\varepsilon_{f 2 y c m}=\infty$ \\
\hline 2 & ПИ-закон & $\varepsilon_{f 1 y c m}=a_{1}$ & $\varepsilon_{f 1 y c m}=\infty$ & $\varepsilon_{f 2 y c m}=0$ & $\varepsilon_{f 2 y c m}=\frac{a_{2}}{K_{U}}$ \\
\hline
\end{tabular}

Возмущение, действующее на вход ОУ, может оказывать различное влияние на величину суммарной установившейся ошибки в зависимости от закона управления $\left(\varepsilon_{f 2 y c m}=0, \varepsilon_{f 2 y c m}=a_{2} / K_{U}, \varepsilon_{f 2 y c m}=\infty\right)$. Таким образом, при анализе САУ $\mathrm{c}$ термоэлектрическим объектом необходимо учитывать влияние как $f_{1}$, так и $f_{2}$. При всех практически применимых случаях, когда $\varepsilon_{f 2 y c m} \neq \infty$, были исследованы показатели качества переходных процессов при исследуемых вариантах САУ (рисунок 7).

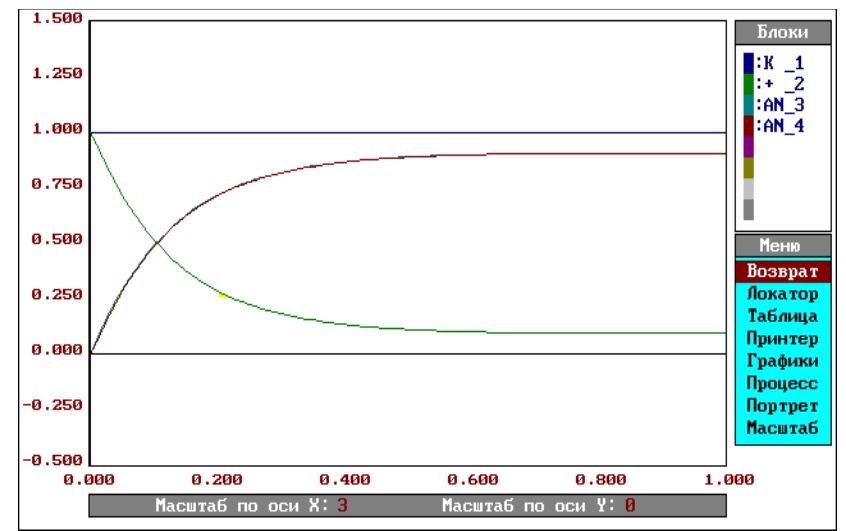

(a)

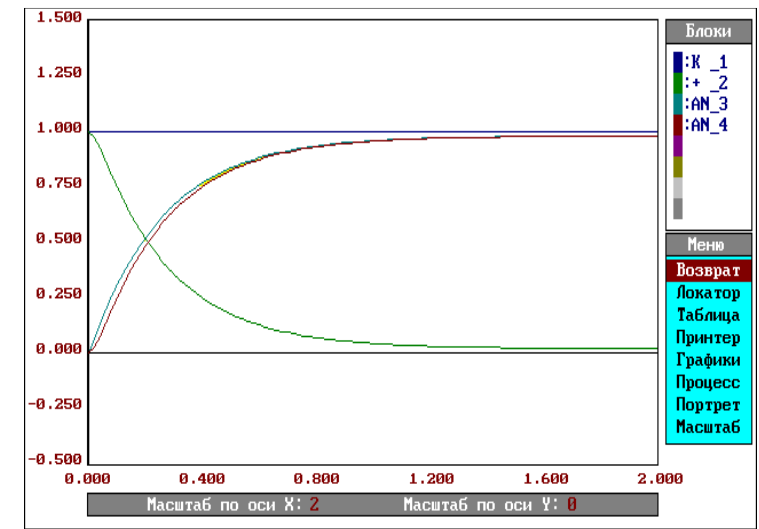

(б)

\section{Рисунок 7 - Анализ показателей качества переходных процессов САУ}

Быстродействие $\left(t_{p}\right)$ и колебательность $(\sigma)$ при указанных значениях коэффициента усиления практически не отличаются от значений, полученных при отсуствии возмущений в САУ (таблица 2). 


\section{Таблица 2 - Значения времени регулирования tр и перерегулирования $\sigma$}

\begin{tabular}{|l|c|c|c|}
\hline & $K_{c}$ & $t_{p}$ & $\sigma$ \\
\hline График (а) & $10 c^{-1}$ & $250 c$ & $0 \%$ \\
\hline График (б) & $50 c^{-1}$ & $60 c$ & $0 \%$ \\
\hline
\end{tabular}

\section{Заключение}

Таким образом, возмущение, действующее на вход САУ, оказывает самое существенное влияния на величину суммарной установившейся ошибки при любом законе управления, поэтому желательно свести ее влияние к минимальнаму, например, компенсировать. Возмущение, действующее на вход ОУ, может оказывать различное влияние на величину суммарной установившейся ошибки зависимости от закона управления (от $\varepsilon_{f 2 y c m}=0$ до $\varepsilon_{f 2 y c m}=\infty$, см. таблица 1$)$.

\section{Список информационных источников}

[1] Ромащев А.А., Арефьев Ю.И. Идентификация и оценивание параметров динамических объектов методом тестовых сигналов // Приборы и системы управления. - 1998. - №.8. - С. 18-19.

[2] Микропроцессорные системы автоматического управления / Под редакцией В.А. Бесекерского. -Л.: Машиностроение, 1988. - 366 с.

[3] Теория автоматического управления. / Под редакцией А.А. Воронова: в 2-х ч. М.: Высшая школа, 1986. Ч.1: Теория линейных систем автоматического управления. - 367 с.

[4] Ни Зо. Использование нечетких регуляторов в системах управления технологическими процессами при производстве полупроводниковых микросхем // Автоматизация и управление в технических системах. - 2012. - № 2; URL: auts.esrae.ru/2-18 (дата обращения: 02.11.2013).

[5] Ни Зо, Николаев А.Б. Моделирование полупроводниковых микросхем с использованием термоэлектрического способа // Автоматизация и управление в технических системах. - 2012. - № 2; URL: auts.esrae.ru/2-19 (дата обращения: 02.11.2013). 